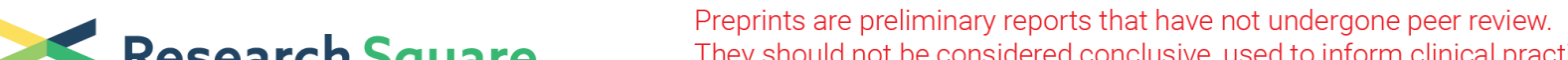 They should not be considered conclusive, used to inform clinical practice, or referenced by the media as validated information.
}

\section{The research of Secretogranin III in periperal blood and vitreous of diabetic retinopathy}

mingfei jiao

Tianjin Medical University Eye Hospital

Shuo Sun

Tianjin Medical University Eye Hospital

Manqiao Wang

Tianjin Medical University Eye Hospital

Lijie Dong

Tianjin Medical University Eye Hospital

\section{Bojie Hu}

Tianjin Medical University Eye Hospital

Juping Liu

Tianjin Medical University Eye Hospital

Wei Li

University of Miami Bascom Palmer Eye Institute

Xiaorong Li ( $\square$ lixiaorong@tmu.edu.cn )

https://orcid.org/0000-0003-0641-2797

\section{Research article}

Keywords: Secretogranin III, periperal blood, vitreous body, vascular endothelial growth factor, diabetic retinopathy

Posted Date: February 12th, 2020

DOl: https://doi.org/10.21203/rs.2.23413/v1

License: (c) (1) This work is licensed under a Creative Commons Attribution 4.0 International License.

Read Full License 


\section{Abstract}

Background: Secretory granulin III (SCG3) is a member of the secretory granule protein family that regulates the production of secretory granules. SCG3 has a role in reducing retinal vascular leakage and neovascularization in an animal model of diabetic retinopathy. This study aimed to study SCG3 in periperal blood and vitreous of human. Methods $\otimes(1)$ Collecting diabetic retinopathy(DR) patients required vitrectomy, and patients requiring vitrectomy for other non-diabetic factors, retaining some of the vitreous, ELISA was used to detect SCG3 in vitreous. The grouping was divided according to the patients' blood lipids, BMI (Body Mass Index, BMI) and analyzed. (2) Collecting peripheral blood of DR and nondiabetic patients, ELISAwas performed. Results: (1) A total of 43 cases with DR were collected, and 34 cases non-diabetic patients were collected. SCG3 in DR patients was higher than that of non-diabetic. After refinement grouping, it was found that SCG3 with DR and hyperlipidemia was higher than that of non-diabetic patients without hyperlipidemia. SCG3 with DR and hyperlipidemia was higher than that of DR patients without hyperlipidemia. SCG3 of patients with DR and high BMI was higher than that of nondiabetic with normal BMI. SCG3 with DR and high BMI was higher than that of non-diabetic patients with normal BMI. (2) SCG3 in plasma was minimal or could not be detected. Conclusion》 In DR patients, upregulation of vitreous SCG3 may be closely related to the pathogenesis of DR. However, SCG3 is almost difficult to detect in normal vessel vasculature, which may highlight its advantages in using anti-SCG3 drugs in babies and children in future.

\section{Background}

Diabetes afflicts 382 million people worldwide and lead to diabetic vascular complications, such as diabetic nephropathy, diabetic neuropathy, and diabetic retinopathy. Vascular endothelial growth factor (VEGF) plays an important role in the pathogenesis of Diabetic macular edema (DME) with retinal vascular leakage and PDR with retinal neovascularization. A breakthrough in DME therapy is the advent and approval of VEGF inhibitors ranibizumab and aflibercept. However, the limited efficacy of VEGF inhibitors suggests that other angiogenic factors may also contribute to the pathogenesis of DR. The challenge is how to identify other DR-related angiogenic ligands for alternative or combination therapy of anti-VEGF DME with high efficacy and safety.

Secretogranin III (Scg3) belongs to the granin family, which consists of chromogranin A ( $\mathrm{CgA}), \mathrm{CgB}$, and Scg2-7. [1] SCG3 is located on chromosome 15q21 and has previously been shown to be associated with obesity [2]. The genetic variation of the SCG3 gene may affect the risk of obesity by regulating the secretion of neuropeptides in the hypothalamus. SCG3 is co-expressed with peptide hormones such as progestin and melanin-concentrating hormone, and forms a granular structure with these neuropeptide hormones. SCG3 was hydrolysised into oligopeptide, which can inhibit food intake and reduce fat accumulation. It has been reported that SCG3 participate in the oxidation process of low-density lipoprotein by participating cells by facilitating the synthesis and secretion of myeloperoxidase. All the researches proved that SCG3 might involved in the process of lipid metabolic diseases. 
Recently, with development of comparative ligandomics, Professor Li Wei [3] did research on DR mouses. Compared with other vascular growth factor, SCG3 is founded performed its function in pathological status. Other vascular growth factor, such as VEGF, which bound to and induced angiogenesis in both diabetic and normal vasculature. Secretogranin III (Scg3) was discovered as a novel disease-associated ligand with selective binding and angiogenic activity in diabetic but not healthy vessels. Scg3 and VEGF signal through distinct and different receptor pathways.

We have previously demonstrated that Scg3 expression was moderately up-regulated by only 1.38-fold, suggesting that increased binding of Scg3 is mainly responsible for its pathogenic role in DR, however, Scg3 expression was not up-regulated in OIR [4]. Considering about this contradictory, we decided to test the concentrations of Scg3 in the peripheral blood and vitrous body of human beings.

\section{Methods}

From May 2018 to December 2018, we collected 77 patients, contained diabetic patients (vitrous hemorrhage and tractional retinal detachment) and non diabetic patients (macular epiretinal membrane patients, macular hole patients, rhegmatogenous retinal detachment, vitrous hemorrhage caused by retinal vascular occlusion, poly choriodal retinopathy). The average age was 31-77 years old, 41 male and 36 female. All the patients were informed the procedures and performed vitrectomy, and all the procedures obeyed the Ethics Committee rules and the declaration of Helsinki. The vitrous body were collected and tested.囚Table 1 ه

\subsection{Inclusion and Exclusion criteria}

Inclusion criteria: All patients met diagnostic criteria and classification of diabetes mellitus in 1999 WHO standards. The fundus examination met diagnostic criteria and guidelines for diabetic retinopathy in China, which was prepared by the Department of Ophthalmology of the Chinese Medical Association Ophthalmology Society in 2014.

All patients were collected detailed medical history and ophthalmology related examinations before surgery, including best corrected visual acuity (BCVA), intraocular pressure, slit exam, ophthalmoscopy, fundus photography, OCT, corneal endothelial cells. Before the operation, the patient should be informed to apply antibiotic eye drops for three days to prevent infection. According to the patient's fundus examination, blood glucose, and the diagnosis of previous diabetes history, it was divided into diabetic retinopathy group and non-diabetic group.

The patient's blood lipids were tested and analyzed. The normal range of total cholesterol was 3.12-5.72 $\mathrm{mmol} / \mathrm{L}$. The normal range of triglycerides is $0.4-1.7 \mathrm{mmol} / \mathrm{L}$. The patients with total cholesterol greater than $5.72 \mathrm{mmol} / \mathrm{L}$ and triglycerides higher than $1.7 \mathrm{mmol} / \mathrm{L}$ were included in the hyperlipidemia group. All the patients were divided into diabetic retinopathy with hyperlipidemia group, diabetic retinopathy with normal lipid group, diabetes with hyperlipidemia group, diabetes with normal lipid group, non-diabetic combined with normal blood lipids group. 
The patient's height and weight were analyzed and the patient's BMI (Body Mass Index, BMI) was calculated. Patients are grouped according to their BMI values. Body mass index = body weight $(\mathrm{kg}) \div$ height squared (m2). BMI between18.5-23.9, normal; BMI between 24-27, overweight; BMI between 28-32, obesity; BMI greater than 32, extreme obesity.BMI of all the enrolled patients was greater than 18.5, between 20.20 to 38.20. All patients were regrouped into diabetic retinopathy with high BMI (PDR+high $\mathrm{BMI}$ ), diabetic retinopathy with normal BMI (PDR+normal BMI), non-diabetic combined with high BMI (non-DM+high BMI), non- Diabetes was combined with the normal BMI group (non-DM + normal BMI).

Exclusion criteria(1) Exclude patients with malignant tumors.(2) Exclude other eye diseases, such as diseases such as history of eye trauma;(3) Exclude pregnancy patients.(4) Exclude patients younger than 18 years old.(5) Exclusion of systemic and ocular infections and fever within 24 hours.(6) Exclude patients with blood system diseases.(7) Routine blood tests, electrocardiogram and other related cardiac function tests were performed on all patients, and exclude patients who are unable to undergo surgery due to other diseases of the system.

\subsection{Surgery and experimental methods}

According to the degree of lens opacity, 35 patients required surgery combined with cataract extraction, and the other 32 patients underwent simple vitrectomy ( 15 patients had been performed cataract extraction surgery previously). The operation is performed by the same surgeon. The eyes were examined before surgery, and both eyes were treated with levofloxacin eye drops to prevent infection. Anesthesia was performed retrobulbal before the operation, with $3 \mathrm{ml}$ lidocaine. Using Constellation Vision System 25G TSV and supporting equipment (Alcon, USA), the cutting rate was $1500 \sim 2000$ times / min, 200 $650 \mathrm{mmHg}$ negative pressure, the intraocular pressure is always maintained at $21 \mathrm{mmHg}$ steadily. The conjunctiva and sclera were slightly misplaced $1-2 \mathrm{~mm}$, the posterior limbus was $4.0 \mathrm{~mm}(3.5 \mathrm{~mm}$ in IOL cases), and a $2 \mathrm{ml}$ syringe was attached to the vitrectomy head. The assistant was used to take the vitreous humor out. The perfusion switch should be closed to prevent the perfusion solution from being mistakenly inserted into the vitreous body, and avoid to affect the accuracy of the data measurement. EP tube is used to collect the specimen, and quickly puts it into the $-80^{\circ} \mathrm{C}$ freezer. ELISA measurements of SCG3 were performed on all vitreous humor. 42 patients with diabetic retinopathy and non-diabetic peripheral blood were collected, plasma was separated, and ELISA was performed.

\subsection{Statistical analysis}

All the experimental datas were statistically analyzed by SPSS 13.0 statistical software. $\unrhd \mathrm{T}$ test $\triangle \mathrm{P}<0.05 \rrbracket$ According to the test of normal distribution, we described continuous variables by median (inter-quartile range) and $\mathrm{N}$ (\%) for categorical variables. The WILCOXON rank-sum test and Chi-square test was applied to compare the difference between genders.

\section{Results}


A total of 43 cases with DR were collected, which conclude 25 cases with hyperlipidemia and 18 cases without hyperlipidemia $₫ 22$ cases with high $\mathrm{BMI}$ and 21 cases with normal BMI. 34 cases non-diabetic patients were collected》which conclude 13 cases with hyperlipidemia and 21 cases without hyperlipidemia $\mathbb{1 7}$ cases with high $\mathrm{BMI}$ and 17 cases with normal BMI.

The concentration of SCG3 of vitreous humor in diabetic retinopathy patients $₫$ median $6.02 \mathrm{ng} / \mathrm{ml} \bigotimes$ interquartile range $7.43 \mathrm{ng} / \mathrm{ml}$ खwas higher than that in non-diabetic patients $\llbracket$ median $4.30 \mathrm{ng} / \mathrm{ml}$ \inter-quartile range7.59 $\mathrm{ng} / \mathrm{ml} \mathbb{\text { Wwhich }}$ was statistically significant $(P=0.019)$. Normality test is a non-continuous variable parameter, after the Man-Whitney test, and there exist significant difference between the two groups. Patients with diabetic retinopathy in the group were stage $\mathrm{V}$ and stage VI. The concentration of

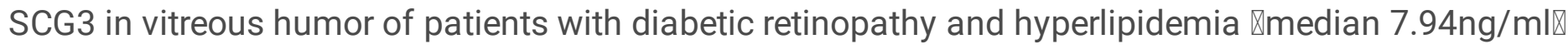
inter-quartile range $8.18 \mathrm{ng} / \mathrm{ml} \rrbracket$ was higher than that of non-diabetic patients without hyperlipidemia \median $4.04 \mathrm{ng} / \mathrm{m}$ \اinter-quartile range $4.65 \mathrm{ng} / \mathrm{ml}$. Normality test is a non-continuous variable parameter, after the Man-Whitney test, with significant statistical difference. $(P=0.001)$. The concentration

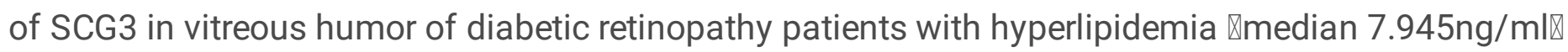
inter-quartile range $8.18 \mathrm{ng} / \mathrm{ml} \mathbb{}$ was higher than that of diabetic retinopathy patients without hyperlipidemia \median $4.45 \mathrm{ng} / \mathrm{ml}$ \inter-quartile range $5.43 \mathrm{ng} / \mathrm{ml}$. Normality test is a non-continuous variable parameter, after the Man-Whitney test, with statistical difference $(P=0.012)$.

The concentration of SCG3 in vitreous humor of patients with diabetic retinopathy and high BMI $₫$ median $7.12 \mathrm{ng} / \mathrm{ml}$ \inter-quartile range $8.56 \mathrm{ng} / \mathrm{ml}$ Wwas higher than that of non-diabetic patients with normal BMI 『median $3.53 \mathrm{ng} / \mathrm{ml}$ \inter-quartile range $1.26 \mathrm{ng} / \mathrm{ml}$. Normality test is a non-continuous variable parameter, after the Man-Whitney test, with statistical difference $(P=0.000)$. The concentration of SCG3 in vitreous humor of patients with diabetic retinopathy and normal BMI 『median 5.72ng/ml囚inter-quartile range7.50 ng/ml $₫$ was higher than that of non-diabetic patients with normal BMI. Normality test is a noncontinuous variable parameter, after the Man-Whitney test, with statistical difference $(P=0.004)$.

42 cases of diabetic retinopathy and non-diabetic peripheral blood were collected, plasma was separated, and plasma enzyme-linked immunosorbent assay was performed. It was found that the concentration of SCG3 in plasma was trace or could not be detected.

\section{Discussion}

Conventional ligand screening methods may neglect disease highly related ligands that have relatively low binding activity to healthy cells $₫$ which has led to the result that SCG3 has never been reported as an angiogenic factor. Ligand proteomics analysis can effectively screen out highly disease related ligands and then perform additional functional analysis. Quantitative comparisons of the entire ligand profile of diseased and healthy cells can comprehensively map all disease-associated ligands, including ligands with increased or decreased binding activity in the states of disease. Prof. Li Wei conducted a study of comparative ligandomics. When applied to diabetic and healthy mice, a total of 353 diabetes mellitus and 105 diabetic low retinal endothelium ligands were systematically identified by comparison of 
histomics. Among a total of 1,772 identified ligands, Scg3 was identied as a DR-high ligand by ligandomics with 1,731 and 0 copies in diabetic and healthy retina, respectively. [4]

Tang [5] et al. conducted a related study on the pathway of SCG3 and found that blocking the MEK/ERK pathway inhibits the expression of SCG3. It was found that SCG3 and VEGF may have different receptor and signaling pathways. SCG3 does not interact with aflibercept, the second binding domain of VEGFR1 fused to third domain of VEGFR2 of human IgG Fc. VEGFR2 is the major receptor for angiogenic signaling [6], but SCG3 fails to activate VEGFR2. SCG3 and VEGF activate Src and may induce vascular leakage by focal adhesion kinase/vascular endothelial cadherin. [7]

Expression of SCG3 was observed in the retina and vitreous humor of diabetic and control mice. Immunohistochemistry showed that SCG3 is expressed in retinal ganglion cells, inner reticulum and outer reticular layers, photoreceptor internal regions and retinal pigment epithelial cells. SCG3 is expressed at similar levels in the homogenate of diabetic and control retinas. SCG3 secretion was confirmed by Western blotting to detect its presence in vitreous humor. SCG3 is significantly upregulated in vitreous humor of 4 month old diabetic mice, suggesting that SCG3 secretion may be a regulated process similar to regulated CTLA-4 secretion [8]. In this study, by measuring the vitreous humor data of the human body, it was found that the content of SCG3 in vitreous humor of patients with diabetic retinopathy was higher than that of non-diabetic patients $(P=0.046)$. It is further confirmed that there may be similar related pathways in human diabetic retinopathy.

SCG3 is a highly disease-associated angiogenic factor with undetectable binding or angiogenic activity in normal blood vessels, however, SCG3 is capable of binding specifically to diseased blood vessels. Perhaps the long-term neglect of SCG3 as a cellular ligand may be due to its very low binding to normal blood vessels and angiogenic activity. The side effects are minimal, which is particularly important for the treatment of retinopathy of prematurity. The treatment is needed to block angiogenesis or angiogenesis in pathological rather than physiological development. Therefore, anti-SCG3 antibodies for the treatment of retinopathy of prematurity can be predicted to be effective and safe due to their disease selectivity.

It has been reported that SCG3 participate in the oxidation process of low-density lipoprotein by participating cells by facilitating the synthesis and secretion of myeloperoxidase. [9] Our experiments have found that patients with diabetic retinopathy and hyperlipidemia have higher levels of vitreous SCG3 than non-diabetic. The concentration of SCG3 in vitreous humor of patients with blood lipids was higher than the control group. Hyperlipidemia might affect the expression and secretion of SCG3. The data showed that the concentration of SCG3 was correlated with diabetes and hyperlipidemia. Hyperlipidemia play an important role in the pathogenesis of SCG3 in PDR patients. In the future, the concentration of SCG3 and high lipoprotein may also be used to detect and assess the severity of diabetic retinopathy.

SCG3 is located on chromosome 15q21 and has previously been shown to be associated with obesity [2]. The genetic variation of the SCG3 gene may affect the risk of obesity by regulating the secretion of 
neuropeptides in the hypothalamus. There is a significant correlation between functional SNP (single nucleotide polymorphism) and obesity in the SCG3 gene. The transcriptional activity of the major alleles, which is higher in the obese subjects than in the normal controls, is lower, suggesting that reduced levels of SCG3 expression may increase the risk of obesity. [10] Even a moderately elevated BMI increases the risk of complications of diabetic retinopathy. [11] The concentration of SCG3 in vitreous humor of patients with diabetic retinopathy and high BMI was higher than that of non-diabetic patients with normal BMI $(P=0.009)$. High BMI may play an important role in the pathogenesis of SCG3 in PDR patients.

It was found that the plasma level of SCG3 was minimal or could not be detected. Undetected SCG3 binding to healthy retinal endothelium may not indicate an absolute deletion of the Scg3 receptor, but rather indicate a relatively low expression. In other areas, SCG3 is tested to help early diagnosis and future disease prognosis. SCG3 is highly expressed in endocrine and neural tissues. [12] It has been reported in prostate cancer research that the study of the mechanism of cell differentiation has revealed the secretion of SCG3 in the peripheral blood of patients with prostate cancer, suggesting that SCG3 can be used as an indicator for detecting the degree of differentiation of tumor cells. For the detection of changes in prostate cancer and future disease prognosis. [13] And the lack of expression of SCG3 in the normal vasculature may highlight its advantage in the future application of anti-SCG3 drugs: that antiSCG3 has less damage to normal tissue organisms, especially in children.

Human specimens are different from animal and cell samples. Animals and cell samples have good homogeneity, but individual differences are large. The current specimens are still insufficient. In future research, it will be considered to increase the sample size and limit the various conditions when collecting specimens.

In diabetic retinopathy patients, up-regulation of vitreous SCG3 was found. Hyperlipidemia and high BMI may play an important role in the pathogenesis of SCG3 in PDR patients. SCG3 is almost difficult to detect in normal vessel vasculature, which may highlight its advantages in using anti-SCG3 drugs in babies and children in future. The anti-SCG3 has less damage to normal tissues, especially in babies and children using safty.

\section{Conclusions}

In DR patients, up-regulation of vitreous SCG3 may be closely related to the pathogenesis of DR. However, SCG3 is almost difficult to detect in normal vessel vasculature, which may highlight its advantages in using anti-SCG3 drugs in babies and children in future.

\section{Abbreviations}

secretogranin III, SCG3; vascular endothelial growth factor, VEGF『diabetic retinopathy, DR; Diabetic macular edema, DME; Body Mass Index, BMI 


\section{Declarations}

\section{Ethics approval and consent to participate}

Tianjin Medical University Eye Hospital ethics committee approved the study

In Tianjin Medical University Eye Hospital囚our Eye Institute collect every patient's residual blood in-patient department and signed the agreement with the patients $₫$ that the residual blood was collected after finished routine blood testing.

\section{Consent for publication}

Applicable

\section{Availability of data and materials}

The datasets used and/or analysed during the current study are available from the corresponding author on reasonable request.

\section{Competing interests}

The authors declare that they have no competing interests

\section{Funding}

The Science\&Technology Development Fund of Tianjin Education Commission for High Education

No. $2018 K J 051$

the role of the funding body in the collection of data

\section{Authors' contributions}

MF Jiao for idea冈lab discussion and paper writing $\llbracket S$ Sun for lab work and discussion, MQ Wang for technical help and data collection, $L J$ Dong fortechnical help and data collection, BJ Hu for technical help and data collection, JP Liu for technical help , W Li for idea and discussion $\otimes X R$ Li for idea and discussion

\section{Acknowledgements}

We thank Pro. Zhang xiaomin for discussion.

\section{References}

[1] Toshiyuki Takeuchi, Masahiro Hosaka. $₫ 2008 \llbracket$ Sorting Mechanism of Peptide Hormones and Biogenesis Mechanism of Secretory Granules by Secretogranin III, a Cholesterol-Binding Protein, in 
Endocrine Cells. Current Diabetes Reviews. 4, 31-38

[2]Baghaei F, Rosmond R, Westberg L, et al. $82003 \otimes$ The CYP19 gene and associations with androgens and abdominal obesity in premenopausal women. Obes Res 11:578 -585

[3] Wei Li, Keith A. Webster, Michelle E. LeBlanc, Hong Tian. (2018) Secretogranin III: a diabetic retinopathy-selective angiogenic factor. Cell. Mol. Life Sci. 75:635-647 https://doi.org/10.1007/s00018017-2635-5

[4] Michelle E. LeBlanc, Weiwen Wang, Xiuping Chen, Nora B. Caberoy, Feiye Guo, Chen Shen, Yanli Ji, Hong Tian, Hui Wang, Rui Chen, and Wei Li. $₫ 2017 \llbracket$ Secretogranin III as a disease-associated ligand for

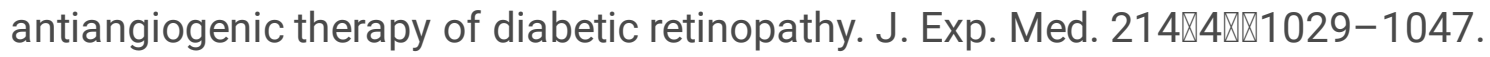

[5] Fen Tang, Mario Thiego F. Pacheco, Ping Chen, Dan Liang, Wei Li. (2018) Secretogranin III promotes angiogenesis through MEK/ERK signaling pathway. Biochemical and Biophysical Research Communications.495®781-786.

[6]Neufeld, G., T. Cohen, S. Gengrinovitch, and Z. Poltorak. 1999. Vascular endothelial growth factor (VEGF) and its receptors. FASEB J. 13:9-22.

[7]Cerani, A., N. Tetreault, C. Menard, E. Lapalme, C. Patel, N. Sitaras, F. Beaudoin, D.Leboeuf, V. De Guire, F. Binet, et al. 2013. Neuron-derived semaphorin 3A is an early inducer of vascular permeability in diabetic retinopathy via neuropilin-1. Cell Metab.18:505-518. doi:10.1016/j.cmet.2013.09.003.

[8]Valk, E., C.E. Rudd, and H. Schneider. 2008. CTLA-4 trafficking and surface expression. Trends Immunol. 29:272-279. doi:10.1016/j.it.2008.02.011

[9]He C, Huang R, Du F, et al. 82009 \&LD oxidation by THP-1 monocytes: Implication of HNP-1, SCG3 and DMT-1. Clin Chim Acta, 402(1-2).102-106.

[10]Tanabe A, Yanagiya T, lida A, et al. 《2007هFunctional single-nucleotide polymorphisms in the secretogranin III (SCG3) gene that form secretory granules with appetite-related neuropeptides are associated with obesity. J Clin Endocrinol Metab. Mar;92(3):1145-54.

[11 ]Gray N, Picone G, Sloan F, Yashkin A.『2015囚Relation between BMI and diabetes mellitus and its complications among US older adults. South Med J. 108(1):29-36. doi:

10.14423/SMJ.0000000000000214.

[12] Chuan Jin, Di Yu, Matko Cancer, et al. (2013)Tat-PTD-Modified Oncolytic Adenovirus Driven by the SCG3 Promoter and ASH1 Enhancer for Neuroblastoma Therapy. HUMAN GENE THERAPY 24:766775.DOI: 10.1089/hum.2012.132.

[13]Jongsma J, Oomen MH, Noordzij MA, et al. $₫ 2002 \bigotimes$ Different profiles of neuroendocrine cell differentiation evolve in the PC-310 human prostate cancer model during long-term androgen deprivation. 
Prostate. 50(4):203-15.

\section{Table 1}

Table 1 Demographic and clinical characteristics of our study sample

\begin{tabular}{ll}
\hline \multicolumn{2}{c}{ clinical characteristics } \\
\hline Total & 77 patients \\
Sex & 41M/36F \\
Age & 31-77 years old \\
diabetic & vitrous hemorrhage and tractional retinal detachment \\
patients & \\
non & macular epiretinal membrane patients, macular hole patients, rhegmatogenous \\
diabetic & retinal detachment, vitrous hemorrhage caused by retinal vascular occlusion, \\
patients & poly choriodal retinopathy \\
& \\
\end{tabular}

\section{Figures}



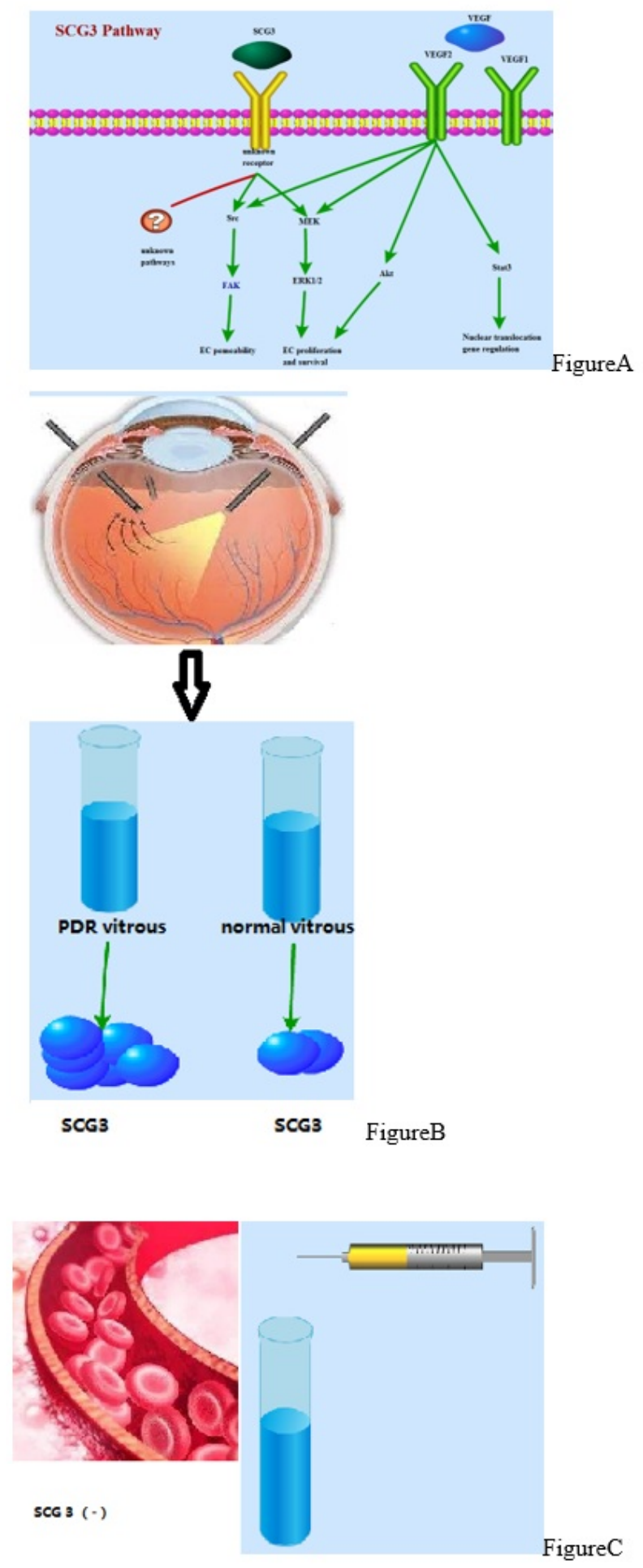

\section{Figure 1}

Scg3 and VEGF receptor signaling pathways; SCG3 in vitreous humor and peripheral blood A The pathway of SCG3 and VEGF: It was found that SCG3 and VEGF may have different receptor and signaling pathways. Scg3 activates ERK and Src pathways, but not Akt and Stat3, through unknown receptors. ERK activated by Scg3 and VEGF may regulate different metabolic events. B The concentration of SCG3 in 
vitreous humor of patients with PDR was higher than that of non-diabetic patients. $C$ It was found that the plasma level of SCG3 was minimal or could not be detected. 